\section{L'Actualité économique}

L'ACTUALITÉ

ÉCONOMIQUE

\section{Inventaire des documents relatifs à l'histoire économique du Canada français - 5}

\section{Cameron Nish}

Volume 44, numéro 1, avril-juin 1968

URI : https://id.erudit.org/iderudit/1003039ar

DOI : https://doi.org/10.7202/1003039ar

Aller au sommaire du numéro

Éditeur(s)

HEC Montréa

ISSN

0001-771X (imprimé)

1710-3991 (numérique)

Découvrir la revue

Citer ce document

Nish, C. (1968). Inventaire des documents relatifs à l'histoire économique du Canada français - 5. L'Actualité économique, 44(1), 176-215.

https://doi.org/10.7202/1003039ar
Ce document est protégé par la loi sur le droit d'auteur. L'utilisation des services d'Érudit (y compris la reproduction) est assujettie à sa politique d'utilisation que vous pouvez consulter en ligne.

https://apropos.erudit.org/fr/usagers/politique-dutilisation/ 


\title{
Inventaire des documents relatifs à l'histoire économique du Canada français $-5^{*}$
}

\author{
1749 \\ C $11 \mathrm{~A}$
}

118. « Memoire sur le convoy du Detroit 1749. (Envoi de vi: vres, marchandises et munitions ; distribution ; canots chargés pour le compte d'individus ; commentaire de Bigot sur l'emploi des canots du Roy.) f 332-333, pp. 92-95.

118. Québec, 31 octobre 1748 «Canada 1748 Depenses a loccasion des mouvements des Pays d'enhaut. Exercice 1749.» (Fonds à remettre en Canada pour les dépenses : à divers individus pour diverses fournitures.) Signé Bigot. f 178-185, pp. 229-244.

118. Miamis, 14 mars 1749. «Certificat. 》 (Gages.) Signé Lanaudière, La Jonquière, Bigot. f 423 , p. 235.

119. Miamis, 6 avril 1749. «Certificat. 》 (Fournitures.) Signé Lanaudière, La Jonquière, Bigot. $f$ 92, p. 86 .

119. Ouyatanons, 7 avril 1749. «Certificat. ». (Fournitures.) Signé Carqueville, La Jonquière, Bigot. f 85 , p. 80 .

118. Détroit, 8 avril 1749. «Fournitures faites par Louis. 》 (Diverses fournitures.) Signé Longueuil, La Galissionière, Bigot. f 357, p. 127.

* On trouvera les autres parties de ce document dans les numéros d'avrilijuin, juillet-septembre et octobre-décembre 1967 ainsi que janvier-mars 1968 de cette revue.

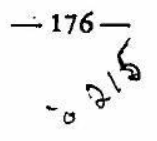


118. Miamis, 10 avril 1749. «Certificat. (Diverses fournitures.) Signé Du Buisson, La Jonquière, Bigot. f 415, p. 227.

119. Ouyatanons, 10 avril 1749. «Certificat.» (Gages.) Signé Carqueville, La Jonquière, Bigot. f 83, p. 79.

118. Miamis, 29 avril 1749. «Certificat. 》 (Diverses fournitures.) Signé Lanaudière, La Galissionière, Bigot. f 409, p. 222.

118. Miamis, 1 mai 1749. «Certificat.» (Gages.) Signé Lanaudière, La Galissionière, Bigot. f 407, p. 220.

118. Miamis, 1 mai 1749. «Certificat.» (Diverses fournitures.) Signé Lanaudière, La Galissionière, Bigot. f 412, p. 225.

118. Miamis, 1 mai 1749. «Certificat.» (Service d'interprète.) Signé Lanaudière, La Jonquière, Bigot. f 439, p. 255.

119. Ouyatanons, 1 mai 1749. "Memoire des fournitures a 8ouattanon. » (Diverses fournitures.) Signé Carqueville, La Jonquière, Bigot. f 80-81, pp. 76-78.

118. Miamis, 4 mai 1749 . « Certificat. 》 (Diverses fournitures.) Signé Lanaudière, La Galissionière, Bigot. f 408, p. 221.

116-1. Versailles, 10 mai 1749. «Pour les depenses de Canada a payer du fonds du Dom'e d'Occident de l'année 1749. » (Officiers de guerre et garnisons entretenus; Québec, Montréal, Trois-Rivières ; maisons religieuses: Québec, Montréal ; officiers de Justice et de Police: Québec, Montréal, Trois-Rivières; autres officiers de Justice; hôpital à Québec; dépenses extraordinaires ; dépenses de l'Isle Royale; dépenses de la Louisianne 1748-1749; récapitulation.) f 208-211, pp. 361-368.

116-1. Versailles, 10 mai 1749. «Pour les Depenses de Canada pendant l'année 1749. » (Construction et radoub de bateaux et canots ; courses et voyages ; fret et voiture ; journées d'ouvriers ; achats de marchandises; présents à faire aux Sauvages ; bois a brûler ; appointements des officiers généraux et autres ; appointements des officiers reformés; solde des compagnies; gages et entretien des employés: Québec, Trois-Rivières, Montréal ; loyers de maisons et bureaux : Québec, Montréal ; gratifications ordinaires, extraordinaires: Québec, Montréal, Trois-Rivières; dépenses pour 


\section{L'ACTUALITE ECONOMIQUE}

préparatifs de guerre ; fortifications et bâtiments civils ; autres diverses dépenses; récapitulation.) f 191-200, pp. 343-360.

119. Ouyatanons, 14 mai 1749. «Memoire des fournitures a 8ouiatanons. 》 (Diverses fournitures.) Signé Carqueville, Poligny, Bigot: f 79 , p. 75.

1.18. Miamis, 15 mai 1749. «Certificat. » (Gages.) Signé Lanaudière, La Jonquière, Bigot. f 406, p. 219.

118. Miamis, 15 mai 1749. «Certificat. » (Fournitures.) Signé Lanaudière, La Galissionière, Bigot. f 417, p. 229.

118. Miamis, 15 mai 1749. «Certificat. 》 (Diverses fournitures.) Signé Lanaudière, La Jonquière, Bigot. f 421, p. 233.

119. Michilimackinac, 16 mai 1749. «Etat de ce qui est du a jean Baptiste Jamin Voyageur pour son payement des vivres qu'il ma fourni dans le Lac Nipissingue. 》 (Diverses fournitures.) Signé Le Gardeur de St. Pierre, La Galissionière, Bigot. f 62-63, pp. 63-64.

119. Ouyatanons, 16 mai 1749. «Memoire des fournitures a uiatanons. 》 (Diverses fournitures.) Signé Carqueville, La Jonquière, Bigot. f 77, pp. 73-74.

119. Ouyatanons, 19 mai 1749. «Certificat. » (Ouvrages aux fusils.) Signé Carqueville, La Jonquière, Bigot. f 76, p. 72.

119. Ouyatanons, 19 mai 1749. «Certificat. » (Gages.) Signé Carqueville, La Jonquière, Bigot. f 82, p. 79.

119. Ouyatanons, 19 mai 1749. «Certificat. » (Fournitures.) Signé Carqueville, La Jonquière, Bigot. f 85, p. 81 .

118. Miamis, 20 mai 1749. «Certificat. » (Fournitures.) Signé La, naudière, La Jonquière, Bigot. f 414, p. 227.

118. Miamis, 20 mai 1749. «Certificat. 》 (Fournitures.) Signé Lanaudière, La Jonquière, Bigot. f 422, p. 234.

119. Miamis, 20 mai 1749. «Certificat. » (Gages.) Signé Lanaudière, La Jonquière, Bigot.' f 97, p. 90.

119. Michilimackinac, 27 mai 1749. « Certificat. ». (Fournitures.) Signé Le Gardeur, La Jonquière, Bigot. f 39, p. 46. 
119. St. Joseph, 28 mai 1749. «Certificat.» (Fournitures.) Signé Piquoté, La Galissionière, Bigot. f 28, p. 36.

119. St. Joseph, 28 mai 1749. «Certificat. 》 (Fournitures.) Signé Piquoté, La Galissionière, Bigot. f 29, p. 36.

119. St. Joseph, 28 mai 1749. «Memoire des Ouvrages que moy Dehestre ay fait a la Riviere St Joseph. » (Divers ouvrages.) Signé Piquoté, La Jonquière, Bigot. f 33, pp. 40-41.

119. Ouyatanons, 20 mai 1749. «Certificat. 》 (Fournitures.) Signé Carqueville, La Jonquière, Bigot. f 84 , p. 80.

118. Miamis, 31 mai 1749. «Certificat. 》 (Gages.) Signé Lanau dière, La Galissionière, Bigot. f 420, p. 232.

118. Miamis, 31 mai 1749. «Certificat. » (Gages.) Signé Lanaudière, La Jonquière, Bigot. f 436, p. 252.

118. Miamis, 1 juin 1749. «Memoire des ouvrages que moi francois Barthe Et Metot ont fait pour le Compte Et service du Roy par l'ordre de Monsieur Delanaudiere Commandant aux miamis. » (Divers ouvrages et fournitures.) Signé Barthe et Metot, Lanaudière, La Jonquière, Bigot. f 425-428, pp. 237-242.

119. Miamis, 1 juin 1749. «Certificat. » (Fournitures.) Signé Lanaudière, La Jonquière, Bigot. f 96, p. 89.

119. Miamis, 1 juin 1749. «Certificat. 》 (Fournitures.) Signé Lanaudière, La Jonquière, Bigot. f 100, p. 91.

118. Détroit, 2 juin 1749. «Memoire des chariages que j'ai faits pour le Roy Sur les demandes du Sr Navarre Tant pour lesbled aux mouliers et au bluteau que pour les autres Effets cy-après Espliquées. 》 (Divers ouvrages et fournitures.) Signé Gamelin, Longueuil, Navarre, La Jonquière, Bigot. f 371, pp. 136-137.

118. Détroit, 4 juin 1749. «Certificat pour Vallée.» (Fournitures.) Signé Navarre, Longueuil, La Galissionière, Bigot. f 344, p. 109.

118. Miamis, 4 juin 1749. «Certificat. » (Fournitures.) Signé Lanaudière, La Galissionière, Bigot. f 413, p. 226. 
118. Miamis, 5 juin 1749. «Cretificat. 》 (Fournitures.) Signé Lanaudière, La Jonquière, Bigot. f 437, p. 253.

119. Miamis, 5 juin 1749. «Certificat. 》 (Fournitures.) Signé La naudière, La Jonquière, Bigot. f 116, p. 105.

118. Détroit, 6 juin 1749. «Memoire des farines qui ont été blutée par Moy Cueillerie, pour le Roy au Detroit Envoyées par le sr Navarre depuis le quatre d'aoust 1747 Jusqu'au 23 d'aoust 1748. » (Farines.) Signé Cuillerier, Navarre, Longueuil, La Jonquière, Bigot. f 370 , p. 135.

118. Miamis, 6 juin 1749. «Certificat. 》 (Gages.) Signé Lanaudière, La Jonquière, Bigot. f 416, p. 228.

118. Miamis, 7 juin 1749. «Fournitures faites par moy Claude Gouin Negociant au Poste des Miamis par L'Ordre de M delanodiere. » (Diverses fournitures.) Signé Claude Gouin, Lanaudière, La Jonquière, Bigot. f 429-432, pp. 243-248.

118. Miamis, 7 juin 1749. «Fournitures par Claude Gouin. » (Diverses fournitures.) Signé Claude Gouin, Lanaudière, La Jonquière, Bigot. f 434, pp. 249-250.

119. Miamis, 7 juin 1749. «Fournitures faites au Roy, suivant le ordres de Monsieur Du Buisson Commandant aux Miamis. » (Diverses fournitures.) Signé du Buisson, La Jonquière, Bigot. f 115 , pp. 103-104.

119. A la Baye, 14 juin 1749. «Fournitures faites par nous fermiers a la Baye aux Nations du Poste. » (Diverses fournitures et bénéfice.) Signé Clignancourt et Compagnie, Verchères, La Jonquière, Bigot. f 15-19, pp. 20-26.

119. A la Baye, 15 juin 1749. «Fournitures faites par nous fermiers de La Baye. » (Diverses fournitures et bénéfice.) Signé Jean Garreau et Compagnies, Verchères, La Jonquière, Bigot. f 12-14, pp. 15-19.

119. A la Baye, 16 juin 1749. «Mémoire de L'ouvrage que moy forgeron ay fait pour le Roy par L'Ordre de Monsieur de Vercheres, Commandant au Poste de la Baye aux nations folles avoines, Puants, Sakis et Renards.» (Divers ouvrages.) Signé Julien, Verchères, La Jonquière, Bigot. f 23-25, pp. 30-34. 


\section{HISTOIRE ECONOMIQUE DU CANADA FRANÇAIS}

119. Michilimackinac, 17 juin 1749. «Certificat. 》 (Fournitures.) Signé Le Gardeur de St. Pierre, La Jonquière, Bigot. f 47, p. 53. 119. Michilimackinac, 18 juin 1749. «Memoire des fournitures que jay fait a Missilimakinac. 》 (Diverses fournitures.) Signé La' joie, Le Gardeur de St. Pierre, La Jonquière, Bigot. f 35, pp. 42-43. 118. Détroit, 20 juin 1749. «Certificat. » (Fournitures.) Signé Navarre, La Galissionière, Bigot. f 345, p. 110.

118. Michilimackinac, 20 juin 1749. «Certificat. 》 (Gages.) Signé Le Gardeur de St. Pierre, La Jonquière, Bigot. f 354, p. 120.

118. Miamis, 20 juin 1749. «Memoire des fournitures faites par Moy Gilbert Parent Negociant au Poste des miamis. 》 (Diverses fournitures.) Signé Parent, Lanaudière, La Jonquière, Bigot. f 435, p. 251.

119. Michilimackinac, 20 juin 1749. «Certificat.» (Vente d'un esclave.) Signé Le Gardeur de St. Pierre, La Jonquière, Bigot. f 38 , p. 46.

119. Miamis, 20 juin 1749. «Certificat. » (Gages.) Signé duBuisson, La Jonquière, Bigot. f 95, p. 89.

119. Miamis, 20 juin 1749. «Certificat. » (Gages.) Signé du Buis` son, La Jonquière, Bigot. f 101, p. 92.

119. Miamis, 21 juin 1749. «Certificat. » (Fournitures.) Signé du Buisson, La Jonquière, Bigot. f 163, p. 151.

119. Détroit, 21 juin 1749. «Certificat. 》 (Pour service d'inter' prète.) Signé Lanaudière, Roy, La Jonquière, Bigot. f 223, p. 213.

118. Détroit, 22 juin 1749. «Mémoire des ouvrages de Menuiseries que Monsieur Le Ch'er de Longueuil Commandant pour Le Roy ai Detroit m'a fait faire Sur le Compte de Sa Majesté. » (Divers ouvrages.) Signé Charles Gariepy fils, Longueuil, Navarre, La Galissionière, Bigot. f 346, p. 111.

119. Miamis, 23 juin 1749. «Certificat. » (Fournitures.) Signé du Buisson, La Jonquière, Bigot. f 99, p. 91.

119. A la Baye, 25 juin 1749. «Certificat. 》 (Fournitures.) Signé Verchères, La Jonquière, Bigot. f 20, p. 25. 
118. Détroit, 28 juin 1749. «Certificat. 》 (Gages.) Signé Lon gueuil, Navarre; La Jonquière, Bigot. f 369, p. 134.

119. Détroit, 28 juin 1749. «Fournitures faites par moy Mantet au Detroit. » (Diverses fournitures.) Signé Navarre, Longueuil, La Jonquière, Bigot. f 230, p. 221.

118. Détroit, 29 juin 1749. «Fournitures faite par Louis Campeau. » (Diverses fournitures.) Signé Longueuil, Navarre, La .Jonquière, Bigot. f 359 , p. 124.

118. Détroit, 29 juin 1749. « Fourniture par Eutrope. » (Diverses fournitures.) Signé Navarre, Longueuil, La Jonquière, Bigot. f 365 , p. 130.

118. Détroit, 29 juin 1749. «Certificat. 》 (Fournitures.) Signé Navarre, Longueuil, Bigot. f 397, p. 209.

118. Détroit, 20 juin 1749. «Etat de plusieurs payemens faits par moy Pierre Vallé au Detroit de Lordre de Monsieur de Longueuol en Pelteries prix du Lieu. » (Divers ouvrages; prix des pelleteries à Détroit et à Québec.) Signé Pierre Vallée, Longueuil, Navarre, La Galissionière, Bigot. f 351, pp, 116-117.

118. Détroit, 30 juin 1749. « Etat des fournitures faites par moy Pierre Reaume au detroit. » (Diverses fournitures.) Signé $\mathrm{Na}$ varre, Longueuil, La Jonquière, Bigot: f 377 , pp. 144145.

119. Michilimackinac, 30 juin 1749. «Etat des fournitures que moi Etinne Dizier Voyageur ai fait pour le Compte du Roy a Missilimakinac. » (Diverses fournitures.) Signé Dizier, Le Gardeur de St. Pierre, La Jonquière, Bigot. f 44, pp. 50-51.

118. Détroit, 1 juillet 1749. «Fournitures faites par Cicot. 》 (Diverses fournitures.) Signé Longueuil, La Jonquière. f 348, p.: 113.

118. Détroit, 3 juillet 1749. «Certificat. » (Gages.) Signé Navarre, Longueuil, La Jonquière, Bigot. f 376, p. 143 .

119. St. Joseph, 4 juillet 1749: «Memoire Des effets que Moy Jean B'te Lefebvre ay fournie a St. Joseph. » (Diverses fournitures.) Signé Jean Baptiste Lefebvre, Piquoté, La Jonquière, Bigot. f 31 , pp. $38-39$. 
119. Michilimackinac, 7 juillet 1749. «Memoire que moy Etienne Dizier a fournit pour le Roy a Missilimakinac. 》 (Diverses fournitures.) Signé Le Gardeur de St. Pierre, La Jonquière, Bigot. f 43, p. 49.

119. Michilimackinac, 8 juillet 1749. «Certificat. » (Gages.) Signé Le Gardeur de St. Pierre, La Galissionière, Bigot. f 48, p. 53.

119. Michilimackinac, 10 juillet 1749. « Estat des traveaux que jay fait au poste de Missilimakinac. » (Divers travaux.) Signé Claude Lesire, Le Gardeur de St. Pierre, La Jonquière, Bigot. f 42, p. 48.

119. Michilimackinac, 10 juillet 1749. «Certificat. 》 (Prix de pension.) Signé Verchères, La Jonquière, Bigot. f 54, p. 58.

119. Michilimackinac, 10 juillet 1749. «Memoire des fournitures fait au Roy pour les folles avoines es Puants Et Sakis qui sont descendu a Montreal. » (Diverses fournitures.) Signé Lajoie, Ver chères, La Jonquière, Bigot. f 59, p. 61 .

119. Michilimackinac, 10 juillet 1749. «Certificat. » (Gages.) Signé duBuisson, La Jonquière, Bigot. f 98, p. 90.

119. Miamis, 10 juillet 1749. «Certificat.» (Gages.) Signé du Buisson, La Jonquière, Bigot. f 119, p. 106.

119. Miamis, 13 juillet 1749. «Certificat.» (Fournitures.) Signé du Buisson, La Jonquière, Bigot. f 118, p. 106.

118. Détroit, 16 juillet 1749. «Memoire Des ouvrages que J'ay faits au Detroit par ordre De Monsieur de Longueuil Pour Le service Duroy, moy Charles Chauvin Taillandrier. » (Divers ouvrages.) Signé Chaivin, Longueuil, Navarre, Bigot. f 399-400, pp. $211 \cdot 213$.

118. Détroit, 16 juillet 1749. «Certificat.» (Vivres.) Signé Navarre, Longueuil, La Jonquière, Bigot. f 404, p. 217.

119. Michilimackinac, 17 juillet 1749. «Memoire des fournitures que j'ay faites pour le Roy par l'ordre de Monsieur de Vercheres, Capitaine pour sa Majesté a la Baye pour donner aux.sauvages dud. Poste. » (Divers fournitures.) Signé Langlade, Verchères, La Jonquière, Bigot. f 11, p. 14. 


\section{L'ACTUALITE ECONOMIQUE}

118. Détroit, 18 juillet 1749. «Certificat. 》 (Gages.) Signé Navarre, La Galissionière, Bigot. f 349, p. 114.

118. Détroit, 18 juillet 1749. «Compte Des bois de chaufage que j'ay Choroyée pour le service du roy suivant les demandes de $\mathrm{Sr}$ navarre Et les ordres de monsieur De longueuil Commandant au Detroit. » (Diverses fournitures, ouvrages et gages.) Signé Mallet, Navarre, Longueuil, Bigot. f 401, p. 214.

119. Michilimackinac, 18 juillet 1749. «Memoire des ouvrages que jay fait. » (Divers ouvrages.) Signé Raymond Menard, Le Gardeur de St. Pierre, La Jonquière, Bigot. f 36, p. 45.

119. Québec, 20 juillet 1749. «Memoire des fournitures que jay faites au Detroit pour ma subsistance dans le voyage a Ouabache. » (Munitions et vivres.) Signé Lanaudière, La Galissionière, Bigot. f 87, p. 82 .

118. Détroit, 21 juillet 1749. «Fournitures par Champagne. 》 (Diverses fournitures.) Signé Champagne, Navarre, Longueuil, La Jonquière, Bigot. f 405, p. 218.

118. Détroit, 24 juillet 1749. «Certificat.» (Gages.) Signé Navarre, Longueuil, La Jonquière, Bigot. f 363, p. 128.

118. Détroit, 24 juillet 1749. «Certificat.» (Gages.) Signé Longueuil, La Jonquière, Bigot. f 368, p. 133.

119. Michilimackinac, 24 juillet 1749. «Certificat.» (Diverses fournitures.) Signé Giasson, Le Gardeur de St. Pierre, La Jonquière, Bigot. f 45, p. 52 .

118. Détroit, 25 juillet 1749. «Memoire des ouvrages Darmurie que j'ay faits pour le service du Roy, moy andré Barthe au Detroit par ordres de Monsieur Le Ch'er de Longueuil Commandant pour le Roy aud. Lieu depuis le Vingt cinq Jullet mil sept cent quarante huit jusqu'a ce jour.» (Divers fournitures et ouvrages.) Signé André Barthe, Navarre, Longueuil, La Jonquière, Bigot. f 380-383, pp. $149 \cdot 188$.

118. Détroit, 25 juillet 1749. «Memoire des ouvrages que j'ai faits pour les fusils que j'ai recommodes pour les Gens de l'escorte en 
ce poste depuis le 20 juillet 1748. » (Divers ouvrages.) Signé Navarre, La Jonquière, Bigot. f 385-390, pp. 189-202.

119. Michilimackinac, 25 juillet 1749. «Certificat.» (Gages.) Signé Le Gardeur de St. Pierre, La Jonquière, Bigot. f 56, p. 69.

119. Michilimackinac, 25 juillet 1749. « Certificat. » (Fournitures.) Signé Le Gardeur de St. Pierre, La Jonquière, Bigot. f 57, p. 59.

118. Détroit, 26 juillet 1749. «Certificat.» (Gages.) Signé Navarre. f 350 , p. 115 .

119. Michilimackinac, 26 juillet 1749. «Certificat. 》 (Loyer de maison.) Signé Le Gardeur de St. Pierre, La Jonquière, Bigot. f 40, p. 47.

119. Michilimackinac, 26 juillet 1749. «Certificat.» (Loyer de maison.) Signé Le Gardeur de St. Pierre, La Jonquière, Bigot. f 41, p. 47.

119. Michilimackinac, 26 juillet 1749. « Certificat. » (Fournitures.) Signé Duplessis Fabert, La Jonquière, Bigot. f 53, p. 57.

119. Détroit, 26 juillet 1749. «Fournitures par Jean Baptiste Go defroy. \(Diverses fournitures.) Signé Godefroy, Navarre, Longueuil, La Jonquière, Bigot. f 228-229, pp. 218-220.

118. Détroit, 27 juillet 1749. «Certificat.» (Fournitures.) Signé Navarre, Longueuil, La Jonquière, Bigot. f 360, p. 125.

118. Détroit, 27 juillet 1749. «Certificat pour Gaudet. » (Somme empruntée de Gaudet pour les gages.) Signé Navarre, La Jonquière. f 361, p. 126.

118. Détroit, 27 juillet 1749. «Certificat.» (Fournitures.) Signé Navarre, Bigot. f 402, p. 215.

119. Miamis, 27 juillet 1749. «Certificat.» (Fournitures.) Signé du Buisson, La Jonquière, Bigot. f 117, p. 105.

119. Michilimackinac, 28 juillet 1749. «Certificat.» (Diverses fournitures.) Signé Duplessis Fabert, La Jonquière, Bigot. f 55, p. 58.

118. Détroit, 30 juillet 1749. «Certificat.» (Gages.) Signé Navarre, Bigot. f 398, p. 210. 
118. Détroit, 31 juillet 1749. «Certificat. » (Pour Parent, menuisier, pour divers 'ouvrages.) Signé Navarre, La Jonquière, Bigot. f 355, p. 121.

118. Détroit, 31 juillet 1749. «Memoire des fournitures faites par moy Gamelin. » (Diverses fournitures.) Signé Eustache Gamelin, Navarre, Longueuil, La Jonquière, Bigot. f 367, p. 132.

118. Détroit, 31 juillet 1749. «Memoire des fournitures faites par moy Gamelin au Detroit. » (Diverses fournitures.) Signé Eustache Gamelin, Navarre, Longueuil, La Jonquière, Bigot. f 367, p. 132.

118. Détroit, 31 juillet 1749. «Certificat. 》 (Gages.) Signé $\mathrm{Na}$ varre, La Jonquière, Bigot. f 392, p. 204.

118. Détroit, 31 juillet 1749. «Certificat.» (Gages.) Signé Navarre, La Jonquière. f 394, p. 206.

118. Détroit, 2 août 1749. «Certificat.» (Fournitures.) Signé Sabrevrois, Navarre, La Jonquière, Bigot. f 374, p. 141.

118. Détroit, 2 août 1749. «Certificat. 》 (Gages.) Signé Navarre, La Jonquière, Bigot. f 375, p. 142.

119. Michilimackinac, 2 août 1749. «Certificat. 》 (Diverses fournitures.) Signé Duplessis Fabert, La Jonquière, Bigot. f 52, p. 56.

118. Détroit, 3 août 1749. «Certificat. 》 (Gages.) Signé Navarre, La Jonquière. f 352 , p. 118 .

118. Détroit, 3 août 1749. «Certificat.» (Gages.) Signé Navarre, La Jonquière. f 403, p. 216.

118. Détroit, 4 août 1749. «Certificat.» (Fournitưres.) Signé Navarre, La Jonquière, Bigot. f 362, p. 127.

119. Miamis, 4 août 1749. « Certificat. ». (Gages.) Signé du Buis son, La Jonquière, Bigot. f 125, p. 112 .

119. Miamis, 4 août 1749. «Certificat. » (Gages.) Signé du Buis son, La Jonquière, Bigot. f 126, p. 112.

118. Détroit, 5 août 1749. "Certificat. » (Emprunt du Sr. Couagne.) Signé Navarre, La Jonquière, Bigot. f 353, 'p. 119. 
118. Détroit, 5 août 1749. «Certificat. 》 (Fournitures.) Signé de Raymond, La Jonquière, Bigot. f 391, p. 203.

118. Détroit, 5 août 1749. «Certificat.» (Fournitures.) Signé de Raymond, La Jonquière, Bigot. f 393, p. 205.

119. Détroit, 5 août 1749. «Certificat. » (Gages.) Signé Navarre, La Jonquière, Bigot. f 226, p. 215.

119. Miamis, 6 août 1749. «Etat des ouvrages que nous metot forgeron Et Bart armurier avons fai pour le Roy par ordre de $\mathrm{M}$ Dubuisson Commandant au fort des Miamis. » (Divers ouvrages.) Signé duBuisson, La Jonquière, Bigot. f 127, pp. 113-114.

119. Détroit, 6 août 1749. «Certificat.» (Fournitures.) Signé Navarre, Sabrevois, La Jonquière, Bigot. f 262, p. 245.

119. Michilimackinac, 10 août 1749. «Certificat. 》 (Fournitures.) Signé Duplessis Fabert, La Jonquière, Bigot. f 49, p. 54.

119. Détroit, 11 août 1749. «Certificat. » (Fournitures.) Signé de Lery, La Jonquière, Bigot. f 258, p. 241.

119. Michilimackinac, 14 août 1749." "Certificat. 》 (Diverses fournitures.) Signé Duplessis Fabert, La Jonquière, Bigot. f 50, p. 55. 119. Détroit, 19 août 1749. «Certificat. 》 (Gages.) Signé de Lery, La Jonquière, Bigot. f 237, p. 228.

119. Miamis, 1 septembre 1749. «Memoire due moy le Comte for geron et Barte armurier avons fait au fort des miamis depuis le 6 aoust 1749. 》 (Divers ouvrages.) Signé duBuisson, La Jonquière, Bigot. f 130, p. 116.

119. Miamis, 4 septembre 1749. «Certificat. 》 (Achat de maison.) Signé de Raymond, La Jonquière, Bigot. f 131, p. 117.

119. Miamis, 4 septembre 1749. «Etat des Marchandises que moy Sancerre commis de Mr de Godrfroy au poste des miamis que j'ay fourny au Roy. » (Diverses fournitures:) Signé du Buisson, La Jonquière, Bigot. f 132, pp. 118-119.

119. Miamis, 4 septembre 1749. « Estat des fournitures de vivres que moy Sancerre Commis de Mr Godefroy au Poste des Miamis, pour la Nouriture de la garnisons que j'ay livrées: » (Diverses vi- 
vres.) Signé duBuisson, La Jonquière, Bigot. f 134136, pp. 120, 123.

119. Miamis, 9 septembre 1749. «Certificat. 》 (Diverses fournitures.) Signé de Raymond, La Jonquière, Bigot. f 148, p. 136.

119. Michilimackinac, 10 septembre 1749. «Certificat. 》 (Diverses fournitures.) Signé Duplessis Fabert, La Jonquière, Bigot. f 169, p. 157.

119. A la Baye, 15 septembre 1749. «facture des Marchandises que Nous fermiers de $\mathrm{La}$ Baye avons fournie pour le service de $\mathrm{Sa}$ Majesté a Chaque Nation du poste pour faire La Paix avec Le soteux et Missourie faute de pourceline par ordre de Monsieur de Millon officier Commandant. » (Aux Folles Avoines; bénéfice; à la femme et au fils du Chef ; bénéfice ; aux Puants, Renards ; bénéfice.) Signé Jean Garreau et Compagnies, Milon, La Jonquière, Bigot. f 209-214, pp. 199-205.

116-1. (Québec), 17 septembre 1749. «Etat des sommes prevenates du produit des postes des pays d'en haut Recûes par $M$ le $M$ 'is de la Galissioniere depuis le $238^{\prime}$ bre tant de ce qui resteoit alors d'effectif et a recouvrer du produit de la ditte année 1748 que de ce qu'il a recû et reste a recouvrer de la presente année 1749 jusqu'a ce jour le 16 7'bre et des paiemens faits sur les dittes sommes pendant la d. tems savoir. 》 (Recette : sur le produit de 1748 : sur le produit de 1749 ; total de la recette. Dépenses : payé à divers pour gages et gratifications.) f 151-161, pp. 269-285.

119. Miamis, 18 septembre 1749. «Certificat. » (Gages.) Signé duBuisson, La Jonquière, Bigot. f 137, p. 124.

119. Michilimackinac, 20 septembre 1749. «Certificat. 》(Diverses fournitures.) Signé Duplessis Fabert, La Jonquière, Bigot. f 170, p. 158.

119. Michilimackinac, 24 septembre 1749. « Certificat. » (Diverses fournitures.) Signé Duplessis Fabert, La Jonquière, Bigot. f 180, p. 170.

119. Miamis, 26 septembre 1749 . « Memoire de ce que j'ay fourny a Mr de Celoron Commandant Le detachement de la Belle Riviere 
a son passage des miamis. » (Diverses fournitures.) Signé Céloron, La Jonquière, Bigot. f 129, p. 115.

119. Miamis, 26 septembre 1749. «Memoire de ce que Moy Claude Leblond ait fourni a $\mathrm{M}$ de Celoron Commandant Le detachement de la Belle Riviere a son passage. » (Diverses fournitures.) Signé Céloron, La Jonquière, Bigot. f 138, p. 125.

119. Miamis, 26 septembre 1749. «Memoire de ce que moy Lo is Clement ay fourni a de Celoron le Detachement dela belle Rivieres a sons oassage. » (Diverses fournitures:) Signé Céloron, La Jonquière, Bigot. f 140, p. 126.

119. Miamis, 26 septembre 1749. «Certificat. (Loyers de cheveaux.) Signé Céloron, La Jonquière, Bigot. f 141, p. 127.

119. Miamis, 27 septembre 1749. « Mémoire des ouvrages que moy Le Comte, Et Barte avons fait pour le convoy de Monsieur de Celoron du 26 septembre 1749. 》 (Divers ouvrages.) Signé Le Comte, Céloron, La Jonquière, Bigot. f 143, pp. 129-130.

119. Michilimackinac, 28 septembre 1749. «Certificat. 》 (Diverses fournitures.) Signé Duplessis Faber, La Jonquière, Bigot. f 174, pp. 163-164.

119. Détroit, 28 septembre 1749. «Certificat. 》 (Gages.) Signé Navarre, Sabrevois, La Jonquière, Bigot. f 238, p. 229.

119. Détroit, 28 septembre 1749. «Certificat. 》 (Gages.) Signé Navarre, Sabrevois, La Jonquière, Bigot. f 240, p. 231.

116-1. Québec, 29 septembre 1749. «La Galissionière à Rouillé. 》 (Envoie compte de recettes et dépenses des postes des pays d'en haut pour les deux années de son commandement). Signé La Galissionière. f 142, p. 256.

119. Détroit, 29 septembre 1749. «Certificat. 》 (Gages.) Signé Navarre, Sabrevois, La Jonquière, Bigot. f 224, p. 214.

119. Michilimackinac, 30 septembre 1749. «Certificat. » (Diverses fournitures.) Signé Duplessis Fabert, La Jonquière, Bigot. f 185, p. 176. 
119. Michilimackinac, 1 octobre 1749. «Certificat. 》 (Diverses fournitures.) Signé Duplessis Fabert, La Jonquière, Bigot. f 172, pp. 160-161.

119. Michilimackinac, 2 octobre 1749: «Certificat. 》(Diverses fournitures.) Signé Duplessis Fabert, La Jonquière, Bigot. f 171, p. 159.

119. Michilimackinac, 2 octobre 1749. «Certificat.» (Diverses fournitures.) Signé Duplessis Pabert, La Jonquière, Bigot. f 175, p. 165 .

119. Michilimackinac, 2 octobre 1749. «Certificat.» (Fournitures.) Signé Duplessis Fabert, La Jonquière, Bigot. f 186, p. 177.

119. Michilimackinac, 2 octobre 1749. «Certificat.» (Fournitures.) Signé Duplessis Fabert, La Jonquière, Bigot. f 192, p. 183.

119. Michilimackinac, 2 octobre 1749. «Certificat.» (Fournitures.) Signé Duplessis Fabert, La Jonquière, Bigot. f 195, p. 185.

119. Michilimackinac, 2 octobre 1749. «Certificat.» (Diverses fournitures.) Signé Duplessis Fabert, La Jonquière, Bigot. f 199, p. 188.

119. Détroit, 2 octobre 1749. «Certificat. (Fournitures.) Signé Navarre, Sabrevois, La Jonquière, Bigot. f 225, p. 214.

119. Michilimackinac, 3 octobre 1749. «Memoire des ouvrages que jay fait par ordre de Monsieur Duplessis commandant pour le Roy a Missilimakinac. 》 (Divers ouvrages et fournitures.) Signé Duplessis Fabert, La Jonquière, Bigot. f 178, pp. 168-169.

119. Michilimackinac, 3 octobre 1749: « Memoire des Vivres dont jay besoin pour mon voyage. » (Diverses vivres.) Signé Le Gar' deur de Repentigny, Duplessis Fabert, La Jonquière, Bigot. f 181, p. 171.

119. Michilimackinac, 3 octobre 1749. «Certificat. 》 (Fournitures.) Signé Duplessis Fabert; La Jonquière, Bigot. f 184, p. 175.

119. Michilimackinac, 3 octobre 1749. «Certificat. \) (Diverses fournitures.) Signé Duplessis Fabert, La Jonquière, Bigot. f 191, p. 182 . 
119. Michilimackinac, 4 octobre 1749. «Certificat. 》(Fournitures.) Signé Duplessis Fabert, La Jonquière, Bigot. f 163, p. 162.

119. Michilimackinac, 4 octobre 1749. «Certificat.» (Gages.) Signé Duplessis Fabert, La Jonquière, Bigot. f 177, p. 167.

119. Michilimackinac, 4 octobre 1749. «Certificat. 》(Fournitures.) Signé Duplessis Fabert, La Jonquière, Bigot. f 198, p. 187.

119. Détroit, 5 octobre 1749. «Fournitures par Godefroy. » (Diverses fournitures.) Signé Navarre, Sabrevois, La Jonquière, Bigot. f 207, pp. 216-217.

119. Détroit, 6 octobre 1749. «Certificat. 》 (Fournitures.) Signé Navarre, Sabrevois, La Jonquière, Bigot. f 253, p. 238.

119. Détroit, 6 octobre 1749. «Certificat. 》 (Pournitures.) Signé Navarre, Sabrevois, La Jonquière, Bigot. f 270, p. 250.

118. Détroit, 20 octobre 1749. "Certificat.» (Gages de Toussignant pour travaux de menuisier.) Signé Longueuil, Navarre, La Jonquière, Bigot. f 356, p. 122.

119. Québec, 22 octobre 1749. "Etat des Munitions et vivres qui ont été delivrés des magasins du Roy a Quebec et Envoyé a chédaik de L'Acadie, tant pour la subsistance et service des officiers et soldats des Troupes et habitants rétachés pour aller aud't lieu, sous le commandement de $\mathrm{Mr}$ De Lacorne capitaine de Compagnies, que pour celle des habitants dud' lieur de L'Acadie qu'il En suit. » (Munitions et marchandises; vivres; sommaire.) Signé Estèbe, Bréard, Bigot. f 3.7, pp. 2.9.

119. Québec, 27 octobre 1749. «Depenses Concernant Le Parti Envoyé a la Riviere St Jean Sous le Commandement du Sr de Boishebert 1749.» (Munitions de guerre et de bouche; vivres; sommaire.) Signé Estèbe, Bréard, Bigot. f 9-10, pp. 10-13.

118. Québec, 29 octobre 1749. «Depenses a l'occasion des Mouvemens des Pays d'enhaut. 》 (Fonds à remettre pour le payement de : divers ouvrages, marchandises et munitions à Détroit, aux Miamis, à Michilimackinac, Rivière St. Joseph, et $\mathrm{A}$ la Baye ; sommaire.) Signé Bigot. f 334-340, pp. 96-108. 
118. Québec, 31 octobre 1749. «Bigot au Ministre. 》 ( $\AA$ propos des dépenses des pays d'en haut. Total de la dépense. Il y a d'autres dépenses qui ont été présentées mais qui ne sont pas comprises dans le présent état. Elles le seront dans le compte de l'an prochain. L'ordre qu'il a donné aux commandants de tenir des journaux les gênera beaucoup. Le Général peut aider beaucoup les choses en mettant des officiers qui ne sont pas de part avec les fermiers. Les officiers ne devront pas faire le commerce. Beaucoup de marchandises sont portées dans les canots du Roy mais pour le service d'au' tres que le Roy.) Signé Bigot. f 327-331, pp. 88-91.

119. Québec, 31 octobre 1749. «Bigot au Ministre.» (Envoi de l'état des munitions; commentaire sur les postes commandés par Boishebert et de la Corne; montant des états.) Signé Bigot. f 2, p. 1.

116-1. Québec, 27 mai 1750. «Dépense concernant le Port de Rocgefort. » (Bordereau de la dépense faite au Canada pendant l'année 1749 pour la construction des vaisseaux du Roy: gages des ouvriers engagés à la journée, au mois; ouvrages: atelier ; achats de marchandises et munitions : bois, mâts, planches, merains, chanvre, cordages, charbon de terre et de bois, fers et clous ; ouvrages de taillandries; ouvrages de serrurerie; diverses marchandises ; fret et voiture ; appointements : officiers de port et de plume ; solde : gardiens et portiers ; hôpitaux : Hôtel-Dieu de Québec ; loyers de maisons, magasins et bureaux; dépenses extraordinaires; sommaire.) Signé Bigot. f 263-271, pp. 13-16.

116-1. Québec, 24 octobre 1750. «Etat des fonds faits par les Etats du Roy du montant des Recettes Extraordinaires et des dépenses faites en Canada sur les Exercices de 1746, 47, 48 et 1749 pour servir a Constater les Excedents de Depenses de chacun de ces Exercices. 》 (Fonds faits par l'état du Roy, 1749 ; recettes extraordinaires ; récapitulation. Dépenses; sommaire ; récapitulation des excédents.) Signé Bigot. f 238-245, pp. 409-424.

116-1 et 116-2. Québec, 24 octobre 1750. «Bordereau des Re cettes et Dépenses faites par le Sr Taschereau Commis en Canada de Mrs Les Tresoriers Generaux de La Marine pendant L'année 1749 ainsy qu'il Ensuit. » (Recettes : fonds ordonnés par l'état du 
Roy ; à déduire les fonds qui ne peuvent servir de fonds pour les dépenses de 1749 : dépenses de 1748 ; recettes extraordinaires: produits des nouveaux droits ; ventes des magasins : Québec, TroisRivières, Montréal ; total de la recette. Dépenses : construction et radoub de bateaux et canots: Québec, Trois-Rivières, Montréal ; courses et voyages : Québec, Trois-Rivières, Montréal ; fret et voiture : Québec, Trois-Rivières, Montréal ; journées d'ouvriers : Qué, bec, Trois-Rivières, Montréal ; achats de marchandises : en France, à Québec, Trois-Rivières, Montréal ; vivres: Québec, Trois-Rivières, Montréal ; achats de bois à brûler : Québec, Trois-Rivières, Montréal ; hôpitaux : Québec, Trois-Rivières, Montréal ; dépenses imprévues : Québec, Trois-Rivières, Montréal, Chambly, St. Fré deric, Frontenac, Niagara, St. Jean ; appointements des officiers généraux et autres; appointements des officiers reformés; solde des compagnies; gages et entretien des employés: Québec, Trois-Rivières, Montréal, Frontenac, Niagara, St. Fréderic ; loyers de maisons et bureaux : Québec, Montréal ; gratifications ordinaires, ex traordinaires: Québec, Montréal, Trois-Rivières; diverses dépenses ; fortifications et réparations : fortifications : Niagara, St. Jean, Sault St. Louis ; dépenses pour les nouveaux chantiers de construction ; dépenses concernant l'Isle Royale ; artillerie : Québec, Montréal ; totale de la dépense.) Signé Bigot. f 272-331, pp. 37-200.

116-2. Québec, 24 octobre 1750. «Balance des fonds ordonnés par les Etats du Roy et Recettes Extraordinaires pour les Exercices de 1746, 1747, 1748 et 1749 avec les Dépenses faites dans la Colonie Sur ces Exercices et du Montant de ces Dépenses avec les remises faites par les Tresoriers généraux au Tresorier particulier de Lad't Colonie, au moyen de l'acquittement des Lettres de changes qu'il a tirés sur Eux en Consequence de ces Depenses. 》 (Fonds ordonnés par les états et ordonnances et recettes extraordinaires : 1746, 1747, 1748, 1749 ; total des fonds. Dépenses : 1746, 1747, 1748, 1749 ; total des dépenses. Récapitulation. Remises faites par les trésoriers généraux au moyen de lettres de change. Sommaire.) Signé Bigot. f 332-335, pp. 201-205.

116-1. Québec, 26 octobre 1750. «Dépense Concernant le Port de Rochefort. » (Pour la construction du vaisseau du Roy.) Signé Bigot. f 262, p. 12. 
116-2. Québec, 28 octobre 1750. « Extrait du fonds a ordonner par su lement pour Le Parfait payement des dépense generales faites en Canada pendant L'année 1749: 》 (Dépenses : à déduire les fonds ordonnés par l'état du Roy ; fonds à ordonnés.) Signé Bigot. f 333, p. 206.

116-1. Québec, 30 octobre 1750. «Depense concernant le Port de Rochefort. » (Pour la construction de la frégate La Diane.) Signé Imbert, Bigot, Bréard. f 260, p. 11.

116-1. Québec, 31 octobre 1750. «Etat des Lettres de Changes Tirées La presente Année sur Monsieur Boucher Tresorier general des Colonies, Par moy soussigné Jacques Imbert, son commis en Canada, pour le payement des Dépenses faites pendant l'année mil sept cent quarante neuf, Toutes datés et payables comme II suit. \(Parfait payement des dépenses de 1749: du 20 octobre au 14 avril, au 15 avril, au 20 avril, au 21 avril, au 22 avril, au 3 mai, au 7 mai ; sommaire du présent état. Parfait payement des dépenses des mouvements des pays d'en haut, 1749: du 13 octobre au ler juillet. Parfait payement des dépenses de l'Isle Royale, 1749 : du 12 octobre au 15 juillet : Parfait payement des dépenses pour la construction des vaisseaux du Roy, 1749 : du 14 octobre au 15 juin. Pour les dépenses sur l'état de 1751 : du 18 octobre au 15 mai, au 14 juin, au 10 juillet. Sommaire. Récapitulation.) Signé Imbert, Bigot, Bréard. f $247-250$ et $251-259$, pp. $425-430$ et $1-10$.

116-1. Québec, 21 octobre 175i. «Bordereau. General de la Re' cette et Depense faittes en Canada pendant. l'Année 1749 Dirigé suivant les Titres Employés dans l'Etat du Roy arrête le 10. may 1749. » (Recettes extraordinaires : Québec : munitions et marchandises des magasins aux troupes; vivres des magasins aux troupes; vivres aux prisonniers; pelleteries des forts Frontenac et Niagara; castors des forts Frontenac et Niagara ; vivres, munitions et marchandises délivrés a Corbin; prix d'une chaloupe vendue; prix des marchandises pour les Forges de St. Maurice; prix des mar chandises pour la construction de l'Orignal; prix des marchandises pour les matelots; rafraîchissements au vaisseau le Leopard; vivres et marchandises au Leopard; fers et goudron vendus; prix des marchandises pour l'équipage de La Diane; prix des marchan- 
dises pour l'Isle St. Jean ; prix des marchandises pour l'Isle Royale; imposition de Québec; recette des droits; aux Trois-Rivières: ventes des magasins du Roy; Montréal : marchandises vendues dans les magasins du Roy; imposition de Montréal. Dépenses générales : total des dépenses ; fonds ordonnés ; revenans bons ; ex cédents ; motif : Québec : construction et radoub des canots et bateaux ; courses et voyages ; fret et voiture ; journées d'ouvriers ; achats de marchandises ; subsistance et remèdes ; dépenses imprévues; appointements des officiers généraux et autres; appointements des officiers réformés; solde des compagnies; gages et entretien des employés: Québec, Trois-Rivières, Montréal ; loyers des magasins et bureaux: Québec, Montréal ; gratifications ordinaires, extraordinaires : Québec, Trois-Rivières, Montréal ; préparatifs de guerre; fortifications et bâtiments civils ; autres diverses dépenses; autres dépenses non compris dans l'état du Roy mais qui seront sur le régis de 1749 ; fortifications et réparations ; récapitulation.) Signé Bigot. f 212-237, pp. 369-408.

116-1. Québec, 12 août 1752. «Bordereau des Recettes et depenses employées dans le compte rendu a Me Jean Baptiste Jacques Boucher trésorier général des colonies pour l'Exercice 1749 par le Sr Imbert, faisant pour la succession de $\mathrm{Mr}$ taschereau vivant Commis en ce pays de Messieurs Les Trésoriers generaux de $\mathrm{La} \mathrm{Ma-}$ rine. » (Recettes : fonds ordonnés par l'état du Roy; fonds or donnés par supplément, 1751 ; recettes extraordinaires: imposition de Québec, de Montréal ; droits du Domaine ; marchandises livrées à l'Isle Royale; ventes des magasins: Québec, Trois-Rivières, Montréal ; Dépenses : contruction et radoub des bateaux et canots : Québec, Trois-Rivières, Montréal ; courses et voyages : Québec, Trois-Rivières, Montréal ; fret et voiture : Québec, TroisRivières, Montréal ; journées d'ouvriers et façons d'ouvrages: Québec, Trois-Rivières, Montréal ; achats de marchandises : en France, à Québec, Trois-Rivières, Montréal ; achats de vivres: Québec, Trois-Rivières, Montréal ; achats de bois à brûler : Québec, TroisRivières, Montréal ; hôpitaux : Québec, Trois-Rivières, Montréal ; dépenses imprévues: Québec, Trois-Rivières, Montréal, Chambly, Frontenac, Niagara, St. Fréderic, St. Jean, détachement à la Belle Rivière ; établissement d'une nouvelle mission à La Rivière de la 
Présentation; gratifications aux officiers ; appointements et solde des troupes : Québec, Montréal ; Isle Royale ; diverses dépenses ; loyers de maisons : Québec, Montréal ; fortifications et réparations : fortifications: Québec ; réparations : Québec, Montréal, Frontenac, Niagara, St. Fréderic, St. Jean, Sault St. Louis ; artillerie : Québec, Montréal.) Signé Bigot. f 163-19.0, pp. 286-342.

Voir aussi : 1748. 116-1. Québec, 1 novembre 1748. «Bordereau... 》f 138 , p. 250.

Voir aussi : 1750. 119. Détroit, 12 avril 1750. « Certificat. »f 239, p. 230 .

Voir aussi : 1750. 119. Détroit, 3 juin 1750. «Certificat. 》 f 257, p. 240.

Voir aussi : 1750. 119. Détroit, 6 juin 1750. «Certificat. 》f 232, p. 223.

Voir aussi : 1750. 119. Détroit, 6 juin 1750 . «Certificat. 》f 236 , p. 227.

Voir aussi : 1750. 119. Détroit, 19 juin 1750. «Certificat. »f 248, p. 232.

Voir aussi : 1750. 119. Détroit, 19 juin 1750. «Etat... $\gg$ f 249, p. 237.

Voir aussi : 1750, 119. Miamis, 25 juin 1750 . «Etat... »f 145-147, pp. 131-135.

Voir aussi : 1750. 119. Miamis, 25 juin 1750 . «Etat... $\gg$ f 155, pp. $142-145$.

Voir aussi : 1750. 119. Détroit, 6 juillet 1750 . « 1749...»f 212 . 215, pp. 206-208.

Voir aussi : 1750. 119. Détroit, 6 juillet 1750. «Etat... » 244 , p. 234.

Voir aussi : 1750. 119. Détroit, 26 août 1750. «Etat... » 233 , p. 224.

Voir aussi : 1750. 119. Détroit, 26 août 1750. «Etat... »f 274, p. 253 . 
Voir aussi : 1748. 116-2. Québec, 26 octobre 1750. «Extrait...» f 334, p. 207.

Voir aussi : 1750. 119. Québec, 3 novembre 1750. «Compte... » f 364370 , pp. $99 \cdot 110$.

\section{0 \\ C $11 \mathrm{~A}$}

119. Miamis, 27 janvier 1750. «Certificat.» (Diverses fournitures.) Signé de Raymond, La Jonquière, Bigot. f 149, p. 137.

119. Miamis, 15 février 1750. «Certificat. 》 (Diverses fournitures.) Signé de Raymond, La Jonquière, Bigot. f 151, p. 139.

119. Miamis, 24 février 1750. «Certificat.» (Diverses fournitures.) Signé de Raymond, La Jonquière, Bigot. f 91, p. 85.

119. Miamis, 2 avril 1750. «Etat de ce quy a Esté fourni par le S Clermond au Compte du Roy. » (Diverses fournitures.) Signé de Raymond, La Jonquière, Bigot. f 158, pp. 147-148.

119. Miamis, 10 avril 1750. «Certificat. » (Fournitures.) Signé de Raymond, La Jonquière, Bigot. f 150, p. 138.

119. Détroit, 22 avril 1750. «fournitures faites par moy Eustache Gamelin. » (Diverses fournitures.) Signé Navarre, Sabrevois, La Jonquière, Bigot. f 239, p. 230.

119. Miamis, 28 avril 1750. «Certificat. 》(Diverses fournitures.) Signé de Raymond, La Jonquière, Bigot. f 152, p. 140.

119. Michilimackinac, 12 mai 1750. «Certificat. » (Diverses fournitures.) Signé Duplessis Fabert, La Jonquière, Bigot. f 189, pp. $18-181$.

119. St. Philippe de Michilimackinac, 17 mai 1750. «Certificat. » (Fournitures.) Signé Duplessis Fabert, La Jonquière, Bigot. f 196, p. 186.

119. St. Joseph, 18 mai 1750. «Certificat. » (Diverses fournitures.) Signé Piquoté, La Jonquière, Bigot. f 90, p. 84.

119. Miamis, 18 mai 1750. «Certificat. 》 (Diverses fournitures.) Signé de Raymond, La Jonquière, Bigot. f 160, p. 149. 


\section{L'ACTUALITÉ ECONOMIQUE}

119. Michilimackinac, 29 mai 1750. « Memoire des fournitures que jay fais moy Blaise Richard par ordre de $\mathrm{M}$ le Commandant d'un canot Envoye pour le service du Roy six sacs de Bled Lessivé a 12 \# le sac. 》 (Diverses fournitures.) Signé Blaise Richard, Duplessis Fabert, La Jonquière, Bigot. f 166, p. 154.

119. Mission de la Présentation, 1 juin 1750. «Etat de la depense que jay faite pour soixante seize sauvages Iroquois establis a la Nouvelle mission de la presentation. 》 (Diverses fournitures.) Signé Piquet, La Jonquière, Bigot. f 276-277, pp. 255-256.

119. St. Philippe de Michilimackinac, 2 juin 1750. «Certificat.» (Diverses fournitures.) Signé Duplessis Fabert, La Jonquière, Bigot. f 193 , p. 184.

119. St. Philippe de Michilimackinac, 2 juin 1750. «Certificat.» (Fournitures.) Signé Duplessis Fabert, La Jonquière, Bigot. f 194, p. 185 .

119. Détroit, 3 juin 1750. «Fournitures faites par Depelteau.» (Diverses fournitures.) Signé Pierre Depelteau, Navarre, Sabrevois, La Jonquière, Bigot. f 257, p. 240.

119. Baye des Puants, 4 juin 1750. «Certificat» (Pour engagement.) Signé de Combre, La Jonquière; Bigot. f 200, p. 189.

119. Baye des Puants, 4 juin 1750. «Certificat. 》 (Pour engage ment.) Signé de Combre, La Jonquière, Bigot. f 201, p. 189.

119. A la Baye, 4 juin 1750. «Memoire des Effets qui ont été fournis par les fermiers du Poste de la Baye pour le service du Roy, Tant aux françois qu'aux sauvages. » (Fournitures et les noms des récipients.) Signé Jean Garreau et Compagnies, de Combre, Bigot. f 204-205, pp. 191-194.

119. Miamis, 6 juin 1750. «Certificat. » (Gages.) Signé de Ray mond, La Jonquière, Bigot. f 93, pp. 86-87.

119. Détroit, 6 juin 1750. «Certificat pour Lamothe. » (Diverses fournitures.) Signé Lamothe, Navarre, Sabrevois, La Jonquière, Bigot. f 232, p. 223.

119. Détroit, 6 juin 1750. «Certificat pour de Quindre. 》(Diverses fournitures.) Signé Dagneau de Quindre, Navarre; Sabrevois, La Jonquière, Bigot. f 236, p. 227. 
119. Détroit, 13 juin 1750. «Certificat pour Pierre Rheaume.» (Diverses fournitures.) Signé Pierre Rhéaume, Navarre, Sabrevois, La Jonquière, Bigot. f 260, p. 243.

119. Miamis, 14 juin 1750. «Certificat. » (Gages.) Signé de Ray॰ mond, La Jonquière, Bigot. f 102, p. 92.

119. Miamis, 15 juin 1750. « Certificat. » (Gages.) Signé de Ray mond, La Jonquière, Bigot. f 103, p. 93.

119. Miamis, 15 juin 1750. «Certificat. 》 (Diverses fournitures.) Signé de Raymond, La Jonquière, Bigot. f 105, p. 95.

119. A la Baye, 15 juin 1750. «Certificat. 》 (Diverses fournitures.) Signé Jean Baptiste Lamonerie, s.j., La Jonquière, Bigot. f 208, p. 198.

119. Miamis, 18 juin 1750. «Certificat. » (Gages.) Signé de Ray• mond, La Jonquière, Bigot. f 106, p. 96.

119. Miamis, 18 juin 1750. «Certificat. » (Gages.) Signé de Ray• mond, La Jonquière, Bigot. f 113, p. 101.

119. Miamis, 18 juin 1750. «Certificat. 》 (Diverses fournitures.) Signé de Raymond, La Jonquière, Bigot. f 142, p. 128.

119. Miamis, 18 juin 1750. «Certificat. » (Gages.) Signé de Ray mond, La Jonquière, Bigot. f 154, p. 141.

119. Miamis, 18 juin 1750. «Certificat. 》 (Diverses fournitures.) Signé de Raymond, La Jonquière, Bigot. f 159, p. 148.

119. Miamis, 18 juin 1750. « Certificat. » (Gages.) Signé de Ray» mond, La Jonquière, Bigot. f 164, p. 152.

119. Miamis, 19 juin 1750. «Certificat. » (Gages.) Signé de Ray mond, La Jonquière, Bigot. f 107, p. 96.

119. Miamis, 19 juin 1750. «Certificat. » (Gages.) de Raymond, La Jonquière, Bigot. f 111, p. 100.

119. Miamis, 19 juin 1750. «Certificat. » (Gages.) Signé de Ray mond, La Jonquière, Bigot. f 112, p. 101.

119. Détroit, 19 juin 1750. «Etat des fournitures faites par moy Charles Chesne au detroit.» (Diverses fournitures.) Signé $\mathrm{Na}$ varre, Sabrevois, La Jonquière, Bigot. f 220, p. 211. 
119. Détroit, 19 juin 1750. «Etat des fournitures faites par Moy Godefroy au Detroit. »(Diverses fournitures.) Signé Godefroy, Navarre, Sabrevois, Bigot. f 241, p. 232.

119. Détroit, 19 juin 1750. «Etat des ouvrages et fournitures faites par moy Louis Campault au Detroit. » (Divers ouvrages et four' nitures.) Signé Louis Campeau, Navarre, Sabrevois, La Jonquière, Bigot. f 249, p. 237.

119. Détroit, 19 juin 1750. «Fournitures faites par moy Pierre Teaume au Detroit. \ (Diverses fournitures.) Signé Pierre Rhéaume, Navarre, Sabrevois, La Jonquière, Bigot. f 259, p. 242.

119. Détroit, 19 juin 1750. «Certificat.» (Fournitures.) Signé Navarre, Sabrevois, La Jonquière, Bigot, f 268, p. 249.

119. Miamis, 20 juin 1750. «Certificat. (Diverses fournitures.) Signé de Raymond, La Jonquière, Bigot. f 104, p. 94.

119. Miamis, 24 juin 1750. «Etat des Effets que nous Loranger et marin Le duc associé avons fourny et delivrés suivant l'ordre de $\mathrm{M}$ le Commandant pour la teste Blanche premier chef des 8aitanon qyi Est venus Luy Cinquieme amener une Esclave pour Monsieur le General et apporté une chevelure chikichiat. » (Diverses fournitures.) Signé de Raymond, La Jonquière, Bigot. f 109, pp. 98-99.

119. Miamis, 25 juin 1750. «Etat des fournitures que moy Claude LeBlond ayt fait pour le Compte et Service du Roy par ordre de M Raymond, Capitaine et Commandant pour le Roy ao fort des miamis. 》 (Diverses fournitures.) Signé de Raymond, La Jonquière, Bigot. f 145-147, pp. 131-135.

119. Miamis, 25 juin 1750. «Etat des vivres en Bleds des gesses que moy Louis Clermond que j'ay esté au poste des ouiatanons que jay fourny et mis dans les Magazins du Roy de ce poste en date du 4 aoust 1749 et la nouriture du detachement de dix homme de cette garnison depuis le 3 avril jusque au 18 de juin. » (Diverses fournitures.) Signé de Raymond, La Jonquière, Bigot, f 155, pp. 142 . 145.

119. Miamis, 25 juin 1750. «Certificat.» (Fournitures.) Signé de Raymond, La Jonquière, Bigot. f 165, p. 153. 
119. Miamis, 27 juin 1750. «Certificat. 》 (Fournitures.) Signé de Raymond, La Jonquière, Bigot. f 108, p. 97.

119. Miamis, 27 juin 1750. «Certificat. » (Fournitures.) Signé de Raymond, La Jonquière, Bigot. f 114, p. 102.

119. Miamis, 27 juin 1750. «Certificat. » (Fournitures.) Signé de Raymond, La Jonquière, Bigot. f 162, p. 151.

119. Miamis, 1 juillet 1750. «Certificat. » (Diverses fournitures.) Signé de Raymond, La Jonquière, Bigot. f 124, p. 111.

119. Montréal, 3 juillet 1750 . «Etat de la dépense que le $\mathrm{Sr} \cdot \mathrm{Ma}$ ziere Negociant a Montreal a faite au mois de juin mil sept cent cinquante par ordre de Monsieur le Général pour l'Equipement de deux canots d'Ecorce de huit places chacun, destines pour porter Les presents a $\mathrm{M}$ Marin a ordre de faire aux differentes nations sauvages qui frequentent Les postes de $\mathrm{La}$ Baye et des Scioux et pour porter un detachement de soldats avec Leurs vivres et les Ustenciles necessaires pour l'Etablissement d'un fort auxd Postes. 》 (Premier canot : vivres et ustenciles; deuxième canot) Signé $\mathrm{Me}^{\prime}$ zière, La Jonquière, Bigot. f 206-207, pp. 195-197.

119. Détroit, 4 juillet 1750. «Certificat. 》 (Fournitures.) Signé Sabrevois, La Jonquière, Bigot. f 219, p. 210.

119. Miamis, 6 juillet 1750. «Certificat. » (Gages.) Signé de Ray mond, La Jonquière, Bigot. f 121, p. 108.

119. Détroit, 6 juillet 1750. «1749, Poste de la Baye : Recapitulation des marchandises et munitions fournies par Jean Garreau et Comp'ie au poste de la Baye en 1749 pour le service du Roy.» (Diverses marchandises.) Signé Navarre, Sabrevois, La Jonquière, Bigot. f 212-215, pp. 206-208.

119. Détroit, 6 juillet 1750. «Certificat pour Desruisseaux. 》(Diverses fournitures.) Signé Desruisseaux, La Jonquière, Bigot. f 217 , p. 209.

119: Détroit, 6 juillet 1750. «Etat des fournitures par moy labutte et Compagnie. » (Diverses fournitures.) Signé Navarre, La Jon' quière, Bigot. f 244, p. 234.

119. Détroit, 6 juillet 1750. «Certificat.» (Gages.) Signé $\mathrm{Na}$ varre, Sabrevois, La Jonquière, Bigot. f 247, p. 236. 
119. Miamis, 7 juillet 1750 . «Certificat. » (Gages.) Signé de Ray mond, La Jonquière, Bigot. f 123, p. 110.

119. Miamis, 8 juillet 1750 . «Certificat. 》 (Gages.) Signé de Ray mond, La Jonquière, Bigot. f 157, p. 146.

119. Détroit, 8 juillet 1750. «Etat des fournitures par moy Metivier. » (Diverses fournitures.) Signé Metivier, Navarre, Sabrevois, La Jonquière, Bigot. f 275, p. 254.

119. Détroit, 11 juillet 1750. «Certificat. 》 (Fournitures.) Signé Navarre, Sabrevois, La Jonquière, Bigot. f 231, p. 222.

119. Miamis, 12 juillet 1750. "Certificat.» (Diverses fournitures.) Signé de Raymond, La Jonquière, Bigot. f 120, p. 107.

119. Miamis, 12 juillet 1750. «Certificat.» (Fournitures.) Signé de Raymond, La Jonquière, Bigot. f 122, p. 109.

119. Détroit, 19 juillet 1750. «Certificat.» (Fournitures.) Signé Navarre, Sabevois, La Jonquière, Bigot. f 246, p. 235.

119. Détroit, 19 juillet 1750. «Certificat. 》 (Fournitures.) Signé Navarre, Sabrevois, La Jonquière, Bigot. f 248, p. 236.

119. Québec, 30 juillet 1750. «Memoire des depenses que j’ay faites pour conduire les nations de la Baye des Puans a Montreal, 207 sauvages, et Cinq François de 4 juin 1750. » (Diverses fournitures et vivres.) Signé de Combre, La Jonquière, Bigot. f 202, p. 190.

119. Québec, 1 août 1750. « Memoire des vivres que moy Reaume Interprette ait été obligé d'acheter a Missilimakinac l'automne dernier pour la subsistance des Sakis, Renards, folavoine Et Puant pour les consuire a leurs villages» (Diverses fournitures.) Signé Jean Baptiste Rhéaume, La Jonquière, Bigot. f 188, p. 179.

119. Détroit, 4 août 1750. «Certificat pour Baptiste Chapotons. 》 (Diverses fournitures.) Signé Navarre, Sabrevois, La Jonquière, Bigot. f 221, p. 212.

119. Détroit, 4 août 1750. «Etat des fournitures faitte par moy St Cosme. » (Diverses fournitures.) Signé St. Cosme, Navarre, Sabre' vois, La Jonquière, Bigot. f 242, p. 233. 


\section{HISTOIRE ECONOMIQUE DU CANADA FRANÇAIS}

119. Michilimackinac, 9 août 1750. «Certificat. 》 (Fournitures.) Signé Le Gardeur de Repentigny, La Jonquière, Bigot. f 187, p. 178.

119. Détroit, 13 août 1750. «Certificat.» (Fournitures.) Signé Navarre, Sabrevois, La Jonquière, Bigot. f 235, p. 226.

119. Détroit, 16 août 1750. «Etat des fournitures faites par moy antoine moison En mon particulier au Poste du Detroit. » (Diverses fournitures.) Signé Navarre, Sabrevois, La Jonquière, Bigot. f 273, p. 252 .

119. Détroit 17 août 1750. «Etat des fournitures faites par moy Louis de l'Isle. » (Diverses fournitures.) Signé Navarre, Sabrevois, La Jonquière, Bigot. f 265, p. 247.

119. Détroit, 26 août 1750. «Etat des fournitures faites par moy Eustache Gamelin. » (Diverses fournitures.) Signé Eustache Ga' melin, Navarre, Sabevois, La Jonquière, Bigot. f 233, pp. 224225.

119. Détroit, 26 août 1750. «Certificat.» (Fournitures.) Signé Navarre, Sabrevois, La Jonquière, Bigot. f 251, p. 238.

119. Détroit, 26 août 1750. «Fourniture faites par Moy Antoine Moison. » (Fournitures.) Signé Navarre, Sabrevois, La Jonquière, Bigot. f 271, p. 251.

119. Détroit, 26 août 1750. «Etat des fournitures faites par moy Eustache Gamelin au Poste du Detroit. »(Diverses fournitures.) Signé Eustache Gamelin, Navarre, Sabrevois, La Jonquière, Bigot. f 274 , p. 253.

119. Détroit, 27 août 1750. «Certificat. 》 (Fournitures.) Signé Navarre, Sabrevois, La Jonquière, Bigot. f 272, p. 251.

119. Détroit, 28 août 1750. «Certificat.» (Fournitures.) Signé Navarre, Céloron, La Jonquière, Bigot. f 222, p. 213.

119. Détroit, 28 août 1750. «Certificat. 》 (Fournitures.) Signé Navarre, Sabevois, La Jonquière, Bigot. f 267, p. 248.

119. Détroit, 28 août 1750. «Certificat. » (Fournitures.) Signé Navarre, Sabrevois, La Jonquière, Bigot. f 269, p. 250. 
119. Détroit, 30 août 1750. «Certificat. 》 (Fournitures.) Signé Navarre, Céloron, La Jonquière, Bigot. f 266, p. 258.

119. Détroit, 1 septembre 1750. «Certificat. » (Fournitures.) Signé Céloron, Navarre, La Jonquière, Bigot. f 218, p. 210.

119. Détroit, 2 septembre 1750. «Certificat. 》 (Fournitures.) Signé Navarre, Céloron, La Jonquière, Bigot. f 261, p. 244.

119. Détroit, 3 septembre 1750. «Fournitures faites par moy Del'Isle. » (Diverses fournitures.) Signé Navarre, Céloron, La Jonquière, Bigot. f 263, p. 246.

119. Miamis, 4 septembre 1750. «Certificat.» (Diverses fournitures.) Signé de Raymond, La Jonquière, Bigot. f 161, p. 150.

119. Québec, 26 octobre 1750. «La Jonquière au Ministre. » (Envoi compte des recettes et des dépenses des postes des pays d'en haut du mois d'août 1749 jusqu'en juin 1750. Excédent de recettes; Demande accroissement de ses appointements.) Signé La Jonquière. f 361-363, pp. 96-98.

119. Québec, 28 octobre 1750. «Etat des depenses faites pour le service du Roy dans Les Pays d'Enhaut a l'occasion des mouve' mens des Nations Sauvages Et qui ont été acquittées La presente année mil sept cent cinquante. » (Au Détroit : Céloron, commandant : à divers particuliers pour diverses fournitures et ouvrages ; Poste de la Baye : Marin, commandant : à divers particuliers pour diverses fournitures et ouvrages ; Poste des Miamis : de Raymond, commandant : à divers particuliers pour diverses fournitures et ouvrages; Poste de Michilimackinac: Duplessis Fabert, commandant : à divers particuliers pour diverses fournitures et ouvrages ; Poste des Chouamignon : Marin fils, commandant : à divers particuliers pour diverses fournitures et ouvrages; Poste des Ouyatanons : Carqueville, commandant : à divers particuliers pour diverses fournitures et ouvrages; Poste de la Rivière St. Joseph : Belestre, commandant : à divers particuliers pour diverses fournitures et ouvrages; Poste de la Présentation : Piquet, missionnaire : au Sr. Piquet. Sommaire.) Signé Bigot. f 278-287, pp. 257-277.

116-2. Québec, 30 octobre 1750. «Etat des Lettres de Change premieres et secondes Tirées sur Mons'r Le Munitionnaire géneral des 
Vivres de la Marine par moi soussigné Jacques Imbert Tresorier de la Marine en Canada pour vivres et rafraichissements fournis aux Equipages des Vaisseaux du Roy pendant leur Sejour en la Rade de ce Port la presente année lesd. Lettres dattés du $10 \mathrm{Oc}$ tobre 1750 et payables au 15 fevrier 1751. » (La frégate Langlesia, La Diane, le vaisseau l'Orignal.) Signé Imbert, Bigot, Bréard. f 367 . 368, pp. $248-249$.

116-2. Québec, 30 octobre 1750. «Etat des Lettres de Change Tirées la présente année sur Monsieur de Boullongne Trésorier générale des Colonies par moi soussigné Jacques Imbert son Commis en Canada pour le payement des Dépenses faites pendant l'année mil sept cent cinquante Toutes datées et payables comme il suit. 》 (Dépenses générales : du 9 juin au 20 avril 1751 ; du 14 et 25 juillet et du 3 août et 1 octobre au 21 avril; du 15 octobre au 22 avril, 24 avril, 26 avril, 3 mai, 4 mai, 5 mai, 6 mai, 8 mai, 10 mai, 11 mai, 12 mai, 2 juin, 3 juin, 5 juin, 7 juin, 8 juin, 12 juin, 1 juillet, 5 juillet, 6 juillet ; sommaire. Pour la construction des vaisseaux du Roy : 1750 : lettres du 13 octobre au 15 mai, 9 juin, 3 juillet ; sommaire. Pour les dépenses de l'Isle Royale : 1 octobre au $17 \mathrm{mai}$, 19 mai, 24 mai, 26 mai, 27 mai ; sommaire. Dépense concernant le port de Brest : lettres du 27 octobre au 20 juin. Récapitulation.) Signé Imbert, Bigot, Bréard. f 337-365, pp. 210-246.

116-2. ${ }^{1}$ « Fonds à faire en conséquence des Traittes contenues au present Bordereau. » (De 1751 sur les fonds de la Marine.) f 366, p. 247.

119. Québec, 3 novembre 1750. «Bigot au Ministre. \(Envoie les états des dépenses des pays d'en haut. Elles font partie du compte de 1750. Il espère que les dépenses ne seront pas aussi élevées l'an prochain.) Signé Bigot. f 88, p. 83.

119. Québec, 3 novembre 1750. «Compte de recette et depenses des postes des Pays d'Enhaut tant affermés qu'Ex $x^{\mathrm{T}} / 2$ loitées par congés, que rend $\mathrm{M}$ Le Marquis de la Jonquiere depuis le compte arreté avec $\mathrm{M}$ le Comte de la Galissioniere jusqu'a la presente année 1750. 》 (Produit des postes affermés : 1749 et 1750 : reçu

r. Pas de date mais ce document a été envoyé avec une lettre du 30 octobre I 750 , f 337.365 , pp. 210.246 . 
de La Galissionière ; Témiskamingue, Baye des Puants, Gamanes tigouya et Michipicoton, Chouagamignon ; produit des postes ex ploités par congés : Michilimackinac, Illinois, Rivière St. Joseph, Baye des Puants, Détroit ; total de la recette. Dépenses : aux off, ciers commandant : Détroit, Miamis, Chouagamignon, Michilimac kinac, Baye des Puants, Camanestigouya, Michipicoton, St. Joseph, Ouyatanons, Montreal ; gratifications : Détroit, Baye des Puants, St. Joseph, Belle Rivière, Miamis, Mer de l'Ouest; distribution aux pauvres, aux veuves, aux officiers, aux orphelins, aux hôpitaux ; récapitulation.) Signé La Jonquière. f 364-370, pp. 99-110.

119. Québec, 26 octobre 1751. «Bordereau Général de la Recette et dépense faittes en Canada pendant l'Année Mil sept cent cin' quante Dirigé suivant les Titres Employés dans l'Etat du Roy Ex pedié sur cet Exercice.» (Recettes : extraordinaires: Québec : ventes de munitions et marchandises, de vivres, de munitions et marchandises au Forges, de vivres au Forges, vente de pelleteries, vente des castors remis au bureau, vente d'une chaloupe, prix des fournitures faites pour la frégate $\mathrm{La}$ Diane, vente de fers à $\mathrm{La}$ Diane, vente de marchandises à La Diane, vente de munitions et vivres à La Diane, vente de clous et fer à la Diane, vente de mar. chandises et munitions pour les goélettes, vente de marchandises et munitions à l'Isle Royale, imposition de Québec et Trois-Rivières; vente de marchandises et munitions, Montréal ; total de la recette extraordinaire. Dépenses : motifs des revenans bon et excédents ; construction et radoub de bateaux et canots; courses et voyages ; fret et voiture ; journées d'ouvriers ; achats de marchandises et munitions; achats de bois à brûler; subsistance et remèdes ; dépenses imprévues; appointements des officiers généraux et autres ; appointements des officiers réformés ; solde des compagnies ; solde de la compagnie de canonniers; gages et entretiens des employés: Québec, Trois-Rivières, Montréal ; loyers de magasins et bureaux: Québec, Montréal ; fortifications et réparations ; gratifications ordinaires, extraordinaires: Québec, Montréal, Trois-Rivières; autres diverses dépenses; autres dépenses au compte de 1750 dont les fonds ne sont pas encore faits ; récapitulation.) Signé Bigot. f 371-395, pp. 112-138. 
119. Québec, 12 août 1752. «Bordereau Sommaire des Recettes et Dépenses de l'Exercice 1750. » (Recettes: de l'Etat du Roy; recettes extraordinaires; total de la recette. Dépenses : construction et radoub des bateaux et canots: Québec, Trois-Rivières, Montréal ; courses et voyages : Québec, Trois-Rivières, Montréal ; fret et voiture : Québec, Trois-Rivières, Montréal ; journées d'ouvriers et façons d'ouvrages: Québec, Trois-Rivières, Montréal ; achats de marchandises et munitions: marchandises: en France, à Québec, Trois-Rivières, Montréal; achats de vivres: Québec, Trois-Rivières, Montréal; bois à brûler : Québec, Trois-Rivières, Montréal ; hôpitaux : Québec, Trois-Rivières, Montréal ; dépenses imprévues: Québec, Trois-Rivières, Montréal, Chambly, Frontenac, Niagara, St. Fréderic, Fort de la Présentation, St. Jean, Sault St. Louis, Lac des Deux Montagnes, Fort de la Prairie, Toronto; appointements des officiers généraux et autres; appointements des officiers reformés; appointements et solde des compagnies : Québec, Trois-Rivières, Montréal ; appointements et gages des employés ; loyers de maisons : Québec, Montréal ; gratifications ordinaires, extraordinaires; autres diverses dépenses; gratifications et entretien pour l'Acadie; achats de maisons; établissements des forges; mouvements des pays d'en haut ; diverses dépenses; fortifications et réparations : fortifications : Québec ; réparations : Québec, Trois-Rivières, Montréal, Chambly, Frontenac, Fort de la Présentation, Soulanges, St. Fréderic ; artillerie : Québec, Montréal; total de la dépense.) Signé Bigot. f 332-360, pp. 40-96.

Voir aussi : 1749. 116-1. (Québec), 17 septembre 1749. «Etat des sommes... 》f 158-161, pp. 280.285.

Voir aussi : 1749. 116-1. Québec, 24 octobre 1750. «Etat des fonds... 》 f 242, p. 418.

Voir aussi : 1748. 116-2. Québec, 26 octobre 1750. «Extrait du fonds... » f 334, p. 207.

Voir aussi : 1751. 119. Québec, 25 octobre 1751. «Compte... »f 400-404, pp. $140 \cdot 150$. 


\section{L'ACTUALITE ÉCONOMIQUE}

\section{1 \\ C $11 \mathrm{~A}$}

119. «Etat des payemems que le Roy veut et ordonne être faits par Mr Jean Baptiste Jacques Boucher, Tresorier Genéral des Colonies, pour les Depenses cy après mentionnées faites et a faire pour le service de Sa Majesté en Canada pendant L'année 1751. 》 (Construction et radoub de bateaux et canots et d'ateliers : journées d'ouvriers à la construction de bateaux; journées d'ouvriers pour le radoub de canots; journées d'ouvriers et façon d'ouvrages dans les magasins; achats de marchandises, vivres et munitions; bois de construction; bois à brûler ; mâts et matteaux ; planches ; chanvre; toiles ; goudron; chandelle; huile; charbon de terre; plomb ; fers et clous ; fournitures pour les bureaux ; diverses mar. chandises ; pour les constructions dans la colonie ; vivres de toutes espèces ; fret et voiture ; appointements des officiers généraux et autres; appointements des officiers reformés; solde des compagnies; solde de la compagnie de canonnier; gages et entretien de divers employés: Québec, Trois-Rivières, Montréal, Frontenac, Niagara, Toronto, Détroit, Fort de la Présentation, St. Fréderic, St. Jean, Chambly ; hôpitaux : Québec, Trois-Rivières, Montréal ; loyers : Québec, Trois-Rivières, Montréal, Ste Thérèse, Lac des Deux Montagnes; dépenses extraordinaires : fortifications ; réparations des bâtiments civils; gratifications ordinaires, extraordinaires : Québec, Trois-Rivières, Montréal ; récapitulation.) f 431444, pp. $185-205$.

119. Québec, 18 octobre 1752. «Bordereau General de la Recette et Depense de 1751. 》(Recettes extraordinaires: Québec, TroisRivières, Montréal ; récapitulation de la recette. Dépenses générales : montant de la dépense ; fonds ordonnés ; revenans bon; excédent; motif : construction et radoub de bateaux et canots; courses et voyages; fret et voiture ; journées d'ouvriers; bois à brûler ; diverses autres marchandises et munitions ; vivres de toutes espèces ; fournitures pour les bureaux ; vivres, munitions et marchandises envoyés de France à la Rivière St. Jean; appointements des officiers généraux et autres; gages et entretien de divers employés : Québec, Trois-Rivières, Montréal ; à divers postes ; solde des compagnies; solde de la compagnie de canonnier; appointe- 
ments des officiers reformés ; loyers de maisons et bureaux : Québec, Trois-Rivières, Montréal, Chambly, Lac des Deux Montagnes; gratifications ordinaires, extraordinaires : Québec, Montréal, TroisRivières ; fortifications, artillerie et bâtiments civils ; diverses dépenses; autres dépenses de 1751 pour lesquelles l'état du Roy n'a pas fait de provisions; dépenses imprévues; dépenses concernant I'Acadie; récapitulation.) Signé Bigot. f 405-429, pp. 151-184.

119. Québec, 25 octobre 1751. «La Jonquière au Ministre. » (Envoie le compte général des postes.) Signé La Jonquière. f 399, p. 139.

119. Québec, 25 octobre 1751. «Compte de Recette et Dépense des postes des pays d'En haut, tant affermés que Exploités par congés que rend $\mathrm{Mr}$ le Marquis de la jonquiere depuis son compte de l'année dern're 1239 9'bre jusqu'a la presente année 1751. 》 (Recettes : excédent de 1750 ; produit des postes affermés; $\mathrm{Ca}$ manestiga, Michipicoton, Chouagamignon, Témiscamingue ; produit des postes exploités par congés : Michilimackinac, Illinois, Rivière St. Joseph, Baye des Puants et des Sioux, Détroit. Dépenses : Dé' troit, Miamis, Ouyatanons, Michilimackinac, Rivière St. Joseph, Chouagamignon, Camanestiga, Michipicoton, Baye des Puants, Mer de l'Ouest, Belle Rivière, Montréal ; payements par gratifications : Détroit, Ouyatanons, Rivière St. Joseph ; distribués aux pauvres ; aux veuves; aux officiers retirés sans pension, aux orphelins; aux hôpitaux; aux religieux et religieuses; récapitulation.) Signé La Jonquière. f 400-404, pp. 140-150.

Voir aussi : 1749. 116-1. Québec, 31 octobre 1750. «Etat de Lettres... »f 255-259, pp. 6-10.

Voir aussi : 1749. 116-1. Québec, 21 octobre 1751. «Bordereau General... 》f. 236-237, p. 407.

Voir aussi : 1749. 116-1. Québec, 12 août 1752. «Bordereau... $1749 \ldots \gg$ f 163 , p. 286.

Voir aussi : 1746. 115-2. Québec, 24 octobre 1751. «Bordereau général... 1746. 》f 328-329, p. 344.

Voir aussi : 1752. 119. Québec, 4 novembre 1752.» «Etat...» f $291-316$, pp. $280-323$ et $1-21$. 


\section{2 \\ C $11 \mathrm{~A}$}

119. Québec, 20 octobre 1751. «Projet de l'Etat du Roy pour les Depenses de la Colonie de Canada pendant L'année mil sept cent cinquante deux. » (Construction et radoub de bateaux et canots; courses et voyages ; fret et voiture ; journées d'ouvriers ; achats de marchandises, vivres et munitions; appointements des officiers généraux ; appointements des officiers reformés ; solde des compagnies; solde de la compagnie de canonnier; gages et entretien de divers employés : Québec, Trois-Rivières, Montréal ; à divers postes; hôpitaux : Québec, Trois-Rivières, Montréal ; loyers de magasins et bureaux : Québec, Trois-Rivières, Montréal, Ste Thérèse, Lac Des Deux Montagnes; gratifications ordinaires, extraordinaires : Québec, Trois-Rivières, Montréal ; fortifications et réparations des bâtiments civils; artillerie ; dépenses extraordinaires ; récapitulation.) Signé Bigot. f 445-455, pp. 206-222.

119. Québec, 4 novembre 1752. «Etat des dépenses faites pour le service du Roy dans les pais d'En haut a l'occasion des mouvemens des Nations Sauvages et qui ont été acquitté la presente année mil sept cent cinquante deux. 》 (Détroit : Céloron, commandant : à divers pour diverses fournitures et ouvrages 1751 et 1752 ; Miamis : de Raymond, commandant : à divers pour diverses fournitures et ouvrages 1751 et 1752 ; Michilimackinac: Duplessis Fabert, commandant : à divers pour diverses fournitures et ouvrages 1751 et 1752; Ouyatanons : de Lignerie, commandant : à divers pour diverses fournitures et ouvrages ; Rivière St. Joseph : Le Gardeur de Repentigny, commandant : à divers pour diverses fournitures et ouvrages ; Népigon : Raimbault de St. Blin, commandant : à divers pour diverses fournitures et ouvrages; Baye des Puants: Marin, commandant: à divers pour diverses fournitures et ouvrages; Chouamignon : Marin fils, commandant: à divers pour diverses fournitures et ouvrages; Sandosket : St. Ours, commandant : à divers pour diverses fournitures et ouvrages; Sault Ste. Marie : le chevalier de Repentigny, commandant : à divers pour diverses fournitures et ouvrages; la Belle Rivière : Joncaire, commandant : à divers pour diverses fournitures et ouvrages; Wabash : Longueuil, commandant: à divers pour diverses fournitures et ouvrages; 
Grande Rivière: Beaujeu, commandant : à divers pour diverses fournitures et ouvrages; sommaire.) Signé Bigot. f 291-316, pp. $280-323$ et 1.21 .

119. Québec, 5 novembre 1752. «Bigot au Ministre. » ( $\AA$ propos des dépenses des pays d'en haut. Elles ont augmentées.) Signé Bigot. f 288-289, pp. $278-279$.

\section{3 \\ C $11 \mathrm{~A}$}

119. Québec, 31 octobre 1752. «Projet de l'Etat du Roy pour les Depenses a faire pour le service de sa Majesté en Canada pendant L'année mil sept cent cinquante trois. » (Achats de vivres et mar' chandises et autres dépenses; construction et radoub de bateaux et canots ; courses et voyages ; fret et voiture ; journées d'ouvriers ; munitions, marchandises, vivres ; bois à brûler ; appointements des officiers généraux et autres; solde des compagnies; solde de la compagnie de canonnier; appointements des officiers reformés; gages et appointements de divers employés: Québec, Trois-Rivières, Montréal ; à divers postes; loyers de maisons, magasins et bureaux : Québec, Trois-Rivières, Montréal, Ste Thérèse, Lac des Deux Montagnes ; gratifications ordinaires, extraordinaires: Québec, Trois-Rivières, Montréal ; fortifications ; artillerie ; diverses dépenses extraordinaires.) Signé Bigot. f 456-463, pp. 223-240.

\section{4 \\ C $11 \mathrm{~A}$}

119. Québec, 12 juillet 1755. «Duquesne au Ministre. 》(Donne le compte des recettes et dépenses des postes des pays d'en haut. Recettes: portées de l'année dernière: Détroit, Michilimackinac, St. Joseph, Illinois, la Baye, Mer de l'Ouest, Témiscamingue, Chaougamignon, Népigon, Lac à la Carpe, Camanestiga, Michipicoton. Dépenses : Détroit, Michilimackinac, Belle Rivière, Fort de la Rivière au Bouf, Mer de l'Ouest, La Baye, Chaougamignon, Népigon, Camanestiga, Michipicoton, St. Joseph, Montréal ; distribu tions des graces du Roy; balance.) Signé Duquesne. f 317-324, pp. $27 \cdot 32 .^{2}$ répétés.

. Il y a erreur dans la pagination. Il y a to pages mais les numéros sont 
119. 19 septembre 1758. « Memoire sur les depenses de Canada. » (Commentaire sur les dépenses du Canada et une récapitulation des dépenses.) f $470-475$, pp. 249-257. ${ }^{3}$

\section{APPENDICE}

1141. «Copie d'une lettre de M le Ch'ev d'Arneville Cap'ne Commandant aux alibamous Ecritte a $\mathrm{M}$ le Sueur Commandant a Tombeché. 》f 136, pp. 12-13.

1141. Québec, 15 octobre 1740. «Beauharnois au Ministre.» (Blainville lui a écrit de Michilimackinac à propos des Renards et Sakis.) f 139, p. 16.

1141. Québec, 16 octobre 1740. «Beauharnois au Ministre. » ( $A$ propos d'une lettre commune écrite avec Hocquart demandant trente habits d'ordonnances.) f 138, p. 15.

114-1. Québec, 16 octobre 1740. «Beauharnois et Hocquart au Ministre. » (Ils rendent compte de la conduite de Le Gardeur de St. Pierre contre les Chicachas. Le Gardeur mérite les bontés du Ministre.) f 140 , p. 17.

1141. Québec, 16 octobre 1740. «Beauharnois au Ministre.» ( $\AA$ propos de Salvage de Trémont pour remplir le poste de garde des postes de Montréal.) f 142-143, p. 18.

1141. Québec, 17 octobre 1740. «Beauharnois au Ministre.》 (Au sujet d'un capitaine de navire qui a reçu comme passager un chirurgien sans permission du gouverneur.) Signé Beauharnois. f 137 , p. 14.

1141. Québec, 16 août 1742. «Préambule de la verification de la Caisse du Domaine d'Occident. »f 205, pp. 89-90.

1141. Québec, 16 août 1742. «Appointement de Nouchet comme Receveur du Domaine d'Occident. » Signé Hocquart. f 234, p. 134.

3. Il n'y a pas d'indication concernant le destinataire ni l'auteur de la lettre. 
1141. Québec, 17 août 1742. «Ordre pour faire l'inventaire des effets du Sr. Cugnet. » Signé Hocquart. f 206, pp. 91-92.

1141. Québec, 17 août 1742. «Deliberations des Creanciers du Sr. Cugnet. » Signé Mounier, Thouron, Havy et Lefebvre, Jehanne, Turpin, Gognet, Payes, Bedout et Jayet, Hocquart. f 235-236, pp. $135-137$.

1141. Québec, 21, 22, 26, 27 août 1742. «Etat de mes Effets actyfs et Passifs. » (Dettes : aux engagés de la traite de Tadoussac; gages de l'équipage de la goélette le St. Etienne; achats pour la traite; à la Compagnie des Indes; marchandises à rendre en nature ; pour mon compte particulier pour l'exploitation de la traite de Tadoussac ; pour l'exploitation des Forges de St. Maurice; récapitulation des dettes passives; total des dettes passives; état de mes effets actifs.) Signé Cugnet, Turpin, Hocquart. f 211-217, pp. 96-116.

1141. Québec, 28, 29, 30 août et le 1 et 3 septembre 1742. «Scellés et Inventaire des effets du Sr. Francois Itienne Cugnet. » (Ordonnance requérant la scellée; nom du débiteur; raisons pour la saisie; liste générale des effèts de Cugnet; liste des créanciers de Cugnet ou de leurs représentants. Inventaire: du magasin; du Poste de la Rivière Moisie; de la bibliothèque ; des meubles; des titres de propriété.) Signé Cugnet, Nouchet, Turpin, Taché, De' vienne, Hiché, Boisseau. f 144204, pp. 19-88.

1141. Québec, 18 septembre 1742. « Hocquart au Ministre. » (Au sujet des affaires de Cugnet.) Signé Hocquart. f 220-229, pp. 121127.

1141. Québec, 28 septembre 1742. « Hocquart au Ministre. 》 (Au sujet des affaires de Cugnet.) Signé Hocquart. f 230, p. 128.

115-2. Québec, 6 novembre 1746. «Varin au Ministre. » (Il n’avait pas l'intention de priver les premiers venus de la concession du Poste de Mecatina. Son intention était seulement de prendre le quart pour lui-même. Remercie le Ministre de ses bontés.) Signé Varin. f 309-310, pp. 317-318.

115-2. Québec, 8 novembre 1746. «Varin au Ministre. 》 (Il a reçu la lettre du 5 mai. Il s'est adressé à Hocquart pour permission 


\section{L'ACTUALITE ECONOMIQUE}

du voyage qu'il a fait. Il n'a rien négligé pour recouvrir les déficits de feu Rocbert, ancien garde-magasin à Montréal. Il suggère que pour épargner de l'argent on pourrait acheter les marchandises en France. Les marchandises du Canada sont de 20 à $25 \%$ plus cher.) Signé Varin. f 305-308, pp. 313-316.

115-2. " "Memoire sur les mesures a prendre pour empescher les Anglois de faire des tentatives et descentes dans la nouvelle fran. ce. 》f 311-313, pp. 319-323.

$118{ }^{5}$ « Etat des postes à garnir en Canada et des troupes nécessaires pour les Garnir. »f 164166, pp. 216-218.

118. Québec, 10 novembre 1748. «La Galissionere au Ministre.» (Au sujet de l'édit concernant les esclaves ennemis qui se sauvent dans la colonie.) Signé La Galissionière. f 167, p. 219.

118. Québec, 26 juin 1749. «La Galissioniere et Bigot au Ministre. 》 (Le traité de paix a été rendu public. Les troupes de l'Isle Royale ont été rassemblées à Québec et vont mettre vóile bientôt. Ils ont fixé les limites de St. Jean. La Galissionière se dispose à partir pour Louisbourg.) Signé La Galissionière, Bigot. f 175-176, pp. 225-228.

118. Québec; 24 octobre 1749. «Memoire de Lery. ». (Liste des terres du Point $\mathrm{G}$ au point $\mathrm{H}$ et les noms des propriétaires.) Signé Lery fils. f 171-173, pp. 221-223.

118: Québec, 31 octobre 1748. «La Jonquiere au Ministre.» (Il envoie le mémoire de Lery.) Signé La Jonquière. f :169, p: 220.

119. Québec, 10 septembre 1751. «Recensement des habitans établis aux environs du fort St. Frederic, suivant la visite qui en a été faites les 2, 3 et 4 septembre par le S. Varin Commissaire de la Marine Ordonnateur à Montreal.». (Tableau.) Signé Varin. f 326, p. 34.

119. Québec, 14 septembre 1751. «Memoire pour servir d'Instruction au S Almain Ecrivain ordinaire de la Marine, faisant fonc: tions de Commissaire dans les Postes du Roy :scitués sur les fron-

4. Cette lettre ne porte pas de date, maiś elle était jointe à la lettre de Chaussegros de Lery du 14 novembre 1746 .

5. Pas de date, mais probablement $\times 748$. 


\title{
HISTOIRE ECONOMIQUE DU CANADA FRANÇAIS
}

tières de Canada voisines de l'accadie. 》S Signé Bigot. f 327-331, pp. 35-39.

119. ${ }^{\circledR}$ «Au sujet des troupes du Canada et les dispositions militaires. 》

\author{
Cameron NISH, \\ professeur à l'Université Sir George Williams \\ et \\ directeur de recherche; \\ Centre de Recherches en Histoire économique \\ du Canada français.
} l'auteur.

6. Ce document ne contient aucune indication concernant le destinataire ni 